\section{EL PROCESO DE CREACIÓN GRUPAL EN LAS ACTIVIDADES ARTÍSTICO-EXPRESIVAS COMO ESCENARIO PARA LA NEGOCIACIÓN Y LA RESOLUCIÓN DE CONFLICTOS}

\author{
O PROCESSO DE CRIAÇÃO GRUPAL NAS ATIVIDADES ARTÍSTICO- \\ EXPRESSIVAS COMO CENÁRIO PARA NEGOCIAÇÃO E A RESOLUÇÃO \\ DE CONFLITOS CP
}

THE GROUP CREATION PROCESS IN BODILY EXPRESSION ACTIVITIES AS A SCENARIO FOR NEGOTIATION AND CONFLICT RESOLUTION C P

doi' https://doi.org/10.22456/1982-8918.105472

iD Inma Canales-Lacruz* <bromato@unizar.es>

(iD) Alba González-Palomares** <agonzalezpa@upsa.es>

(iD) Glòria Rovira*** <grovira@euses.ca>

\footnotetext{
*Universidad Zaragoza. Zaragoza, España.

**Universidad Pontificia de Salamanca. Salamanca, España.

***Universidad de Girona. Girona, España.
}

\begin{abstract}
Resumen: Este artículo identifica los factores de la interacción social que facilitan y dificultan el proceso de creación grupal para la elaboración de una composición escénica. La muestra estuvo formada por 105 estudiantes universitarios -85 hombres y 20 mujeres- con una media de edad de 19,99 \pm 2,78 años del Grado de Ciencias de la Actividad Física y el Deporte en EUSES, Universidad de Girona (España). Cada grupo elaboró un informe sobre el proceso de creación en el que reflejaba las dificultades y facilidades ante dicha composición durante las sesiones prácticas de la asignatura Danza y Expresión Corporal. Posteriormente se realizó el análisis de contenido de dichos informes grupales. Para el tratamiento de los datos se utilizó el Nvivo 11. Los resultados mostraron que: a) la interacción social facilita el proceso de creación grupal; b) el consenso es el principal factor de la interacción social que facilita el proceso de creación grupal.
\end{abstract}

Palabras clave: Baile. Consenso. Relaciones interpersonales. Creatividad.
Recibido en: 18-07-2020 Aprobado en: 18-11-2020 Publicado en: 30-12-2020

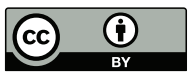

Este es un artículo publicado en Open Access bajo la licencia Creative Commons Atribución 4.0 (CC BY 4.0).

elSSN: 1982-8918 


\section{INTRODUCCIÓN}

\subsection{EL CONFLICTO EN EL SISTEMA EDUCATIVO}

Una de las teorías sobre el conflicto social con mayor renombre parte del funcionalismo estructural, más concretamente de Dahrendorf, el cual, estableció el dualismo conflicto-consenso (SERRAT, 2002). Nuevas teorías como la de Molina (2005) acuñan el término net-socialización para referirse a la nueva socialización globalizada de una sociedad red que tiende a la homogeneidad, pero que también provoca un conflicto ante la necesidad de la singularidad y el localismo. Ante este nuevo horizonte, Molina (2005) propone enfrentarse a esta nueva concepción social desde la inclusión de las habilidades sociales tales como la negociación y el diálogo para la resolución de los conflictos en el sistema educativo.

De hecho, la incorporación de la resolución de conflictos en el sistema educativo es motivo de reflexión por Rojas-Ruiz y Alemany-Arrebola (2016) a partir de los resultados extraídos en una investigación realizada en alumnado universitario. Los resultados mostraron la percepción y conceptualización negativa que tenía el alumnado sobre la temática del conflicto. Además, se constató la escasa concreción de las competencias relacionadas con la temática del conflicto en las guías docentes de las materias de los grados de magisterio.

Otra investigación realizada en el ámbito de la educación física escolar, reveló un alto índice de conflictividad, más concretamente, el $62 \%$ de los juegos motores analizados suscitaron algún tipo de conflicto motor (SÁEZ DE OCÁRIZ; LAVEGA, 2013). Ante dichos resultados, estos autores abogan por la aplicación del índice de conflictividad en los juegos motores y así poder subsanar e introducir estrategias para transformar los conflictos.

Este alto grado de conflictividad en los contenidos de educación física también ha sido constatado por Capllonch, Figueras y Castro (2018). En este caso, la presencia del conflicto fue registrado a partir de las percepciones del propio alumnado, vinculándolo con los modelos más tradicionales de educación física que se basan en juegos competitivos y eliminatorios, desencadenándose conductas de exclusión, enfrentamiento, marginación y conflicto. También Camacho-Miñano y Prat (2018) ponen de manifiesto la violencia simbólica ejercida por una educación física fundamentada en el rendimiento.

Ante esta necesidad de intervención se han incorporado programas y acciones educativas para el desarrollo de las habilidades sociales. Siguiendo con el ámbito de la educación física, más concretamente en la formación universitaria, Arribas-Garralaga et al., (2019) pusieron en práctica una intervención docente basada en proyectos enmarcada en el aprendizaje colaborativo en la asignatura de expresión corporal y comunicación. Los resultados mostraron el aumento de la cooperación, autonomía e implicación del alumnado en los procesos de enseñanza-aprendizaje.

Otros programas de intervención aplicados desde la educación física sobre la prevención y resolución de conflictos, constatan la mejora de la competencia social y ciudadana del alumnado (MONZONíS, 2015). También Capllonch, Figueras y Castro (2018), fomentan la prevención y resolución del conflicto con la incorporación 
de trabajo cooperativo y aprendizaje dialógico. Otras propuestas se abordan desde el trabajo emocional, y es que los contenidos de educación física vinculados con la cooperación y la oposición desencadenan emociones que deben de ser analizadas para fomentar la mejora de la socialización (MOLINA, 2012).

Desde otros ámbitos diferentes a la educación física también han considerado la necesidad de incluir programas de trabajo cooperativo. Por ejemplo, Moll et al. (2019) aplicaron un programa de trabajo cooperativo en la formación universitaria de enfermería. Tras la intervención, la investigación constató la mejora en el proceso de toma de decisiones vinculadas con los requerimientos del trabajo en grupo. Por su parte, Pérez-Albarracín y Fernández-Baena (2019) analizaron la experiencia de estudiantes mediadores en la adquisición de aprendizajes socio-emocionales, registrándose mejoras en la empatía, neutralidad, pensamiento alternativo y la importancia de pedir ayuda. Es decir, el alumnado mediador aprendió a analizar los conflictos de forma aséptica y pensar en diferentes alternativas para solucionar los conflictos.

\subsection{LA INTERACCIÓN SOCIAL EN LOS PROCESOS DE CREACIÓN GRUPAL}

Las tareas artístico expresivas -o también denominadas de expresión corporalse caracterizan porque estimulan la creatividad motriz, y por lo tanto, fomentan el trabajo de la singularidad, requiriendo para ello superar estereotipos motrices. Esta exigencia emocional de ruptura con los patrones de ejecución desencadena nuevas formas de procesar la información, y por lo tanto, genera nuevos escenarios para los procesos de enseñanza-aprendizaje (ARIZCUREN-BLASCO, 2019).

En este sentido, el alumnado muestra falta de práctica al enfrentarse a los procesos de creación, ya que, el miedo a la inexistencia de un modelo paraliza la fluidez de respuestas (Sierra, 2002). De hecho, para contrarrestar dichas carencias, Giguere (2011) defiende la necesidad de plantear propuestas pedagógicas creativas de pensamiento grupal (GIGUERE, 2011) que pongan de manifiesto los beneficios cognitivos como las estrategias comunicativas (BANNERMAN, 2008), los lazos de colaboración (ZANDER et al., 2014) y convivencia (SÁEZ DE OCÁRIZ et al., 2017).

Cuando las propuestas de creación se realizan en grupo se genera sentimiento comunitario, dinámicas de relaciones positivas y la mejora de la eficacia individual y colectiva (REMESAL; COLOMINA, 2013). Además, el grupo no es una entidad rígida, por lo que la red social que se establece se va modificando y reajustando en función de los intereses grupales. Es decir, no se puede olvidar que los procesos de aprendizaje ocurren en un contexto social, lo que enfatiza las dimensiones centradas en el grupo (REMESAL; COLOMINA, 2013).

La colaboración entre los compañeros/as en los diseños de danza de pensamiento grupal se manifiesta de varias formas, siendo la adquisición del rol de facilitador el que apareció en todos los participantes del estudio de Giguere (2011). En esta misma línea de pensamiento grupal, Rymer (2017) planteó un proceso colaborativo e interdisciplinar entre un coreógrafo y un compositor musical, obteniendo satisfacción en ambos independientemente del resultado final. Los resultados demostraron que los requerimientos sociales para esta colaboración creativa fueron la confianza, escucha, comunicación, apertura y la asunción de riesgos. Además, este estudio 
también demostró que la creatividad actúa sobre las posibles diferencias personales consolidando las interacciones sociales.

Otro diseño de pensamiento grupal dirigido a estudiantes universitarios sin experiencia previa en expresión corporal y danza, estableció la importancia del consenso entre los miembros del grupo para compensar las divergencias creativas (TORRENTS et al., 2008). Por su parte, un estudio con bailarinas adolescentes que relacionó el clima motivacional y el bienestar psicológico, sugirió que los profesores de danza deben de ser conscientes del impacto que el clima social ejerce en el bienestar de los bailarines (STARK; NEWTON, 2014). Los resultados mostraron que los climas sociales positivos se relacionan con un mejor afecto, autoestima y relaciones con el profesorado y compañeras. Es por ese motivo que las autoras recomiendan promover tareas que fomenten la colaboración y restar énfasis en la orientación al ego.

Además, otros diseños aplicados en educación superior han determinado la importancia de la interacción social en la creación grupal (HAN; HAN; BRASS, 2014; LIU, 2017). Cuando se gestiona adecuadamente la diversidad de capital humano en los equipos puede ser beneficiosa para la creatividad, ya que, la variedad de conocimiento genera un efecto de unión en el capital social del equipo, es decir, el capital social interfiere en los efectos de la diversidad de conocimiento en la creatividad del equipo (HAN; HAN; BRASS, 2014). Estas investigaciones parten de la base que la creatividad está íntimamente vinculada con el intercambio de conocimiento, con la cultura cooperativa y las relaciones interpersonales.

Otro factor a tener en cuenta en los procesos de creación grupal es la aceptación de las normas de comportamiento social. Las percepciones de comportamiento de quienes nos rodean proporcionan pistas sobre cuál debe de ser el comportamiento, es decir, se generan unas normas grupales que condicionan la conducta social (SHEPHERD, 2017). Esta misma autora sostiene que el cumplimiento de las normas sociales está vinculado con su nivel de visibilidad, y por lo tanto, cuanto mayor sea la percepción de que los miembros del grupo participan en algún comportamiento, mayor será la percepción de la norma descriptiva grupal para esa conducta.

Este trabajo pretende conocer los factores de la interacción social percibidos por el alumnado que dificultan y facilitan los procesos de creación grupal en las sesiones prácticas de un programa de intervención de expresión corporal y danza que se fundamenta en los procesos de creación grupal. Los procesos de creación grupal pueden ser un adecuado escenario para trabajar la negociación y la resolución de conflictos, habilidades sociales que deben de ser desarrolladas e incluidas en el sistema educativo.

\section{MÉTODO}

\subsection{PARTICIPANTES}

La población objeto de estudio de la investigación estuvo constituida por 106 estudiantes universitarios de la asignatura Danza y Expresión Corporal del primer curso del grado de Ciencias de la Actividad Física y del Deporte en la Escuela Universitaria de la Salud y el Deporte -EUSES-, Universidad de Girona -España-. 
La muestra seleccionada fue intencionada de carácter no-aleatorio y estuvo formada por 105 sujetos -85 hombres y 20 mujeres- de 18 a 32 años; $M=19.99$, DT $=2.78$.

El criterio de selección de la muestra fue el grado de participación en las sesiones prácticas de la asignatura. Solamente las personas que siguieron la actividad de forma continuada -asistencia a más del $80 \%$ de las 30 sesiones - podían tener una visión integral de la asignatura. Fue excluido un alumno por falta de participación en las sesiones prácticas.

Los participantes no tenían experiencias previas en actividades artístico expresivas. Se dejó total libertad al alumnado para configurar los grupos para realizar el proceso de creación.

\subsection{VARIABLES}

La variable independiente es el proceso de creación grupal (ROVIRA, 2019), basado en el seguimiento de cuatro etapas, y realizado durante un periodo de dos meses (Cuadro 1). La variable dependiente es la interacción social como factor que facilita o dificulta el proceso de creación grupal en las sesiones prácticas de la asignatura.

Cuadro 1 - Fases del proceso de creación grupal (ROVIRA, 2019)

\begin{tabular}{|c|c|}
\hline & PROCESO DE CREACIÓN GRUPAL \\
\hline $\begin{array}{l}\text { - Objetivo: crear una cc } \\
\text { - Duración: de } 6 \text { a } 12 \text { n } \\
\text { - Composición sin pala } \\
\text { y coreográficos. } \\
\text { - Se transmite un mens } \\
\text { - Proceso que sigue cu } \\
\text { FASE I: Lluvia de ideas } \\
\text { FASE II. Composición } \\
\text { FASE III: Retoque } \\
\text { FASE IV: Muestra final }\end{array}$ & $\begin{array}{l}\text { tosición grupal } \\
\text { tos. } \\
\text { s, es posible usar sonidos corporales y vocales. Recursos de dramatización } \\
\text { a un público. Se busca emocionar al público. }\end{array}$ \\
\hline FASE I: Lluvia de id & \\
\hline $\begin{array}{l}\text { - Nos situamos en el e } \\
\text { - Procuramos los medi } \\
\text { tiempo del que se dis } \\
\text { - Se trata de una fase } \\
\text { - Utilizamos la improvis } \\
\text { - Es fundamental la es } \\
\text { - Partimos de una lluvi } \\
\text { - Consiste en un proce } \\
\text { - Consenso entre los }\end{array}$ & $\begin{array}{l}\text { rno o ambiente adecuado para crear. } \\
\text { adecuados para crear, ya sea por los objetos utilizados, las músicas, el } \\
\text { ne. } \\
\text { loración libre de juicio y de censura. Nos atrevemos a ser y hacer. } \\
\text { ión para explorar posibilidades a partir de objetos, temas diferentes, ideas. } \\
\text { ha, estar atento a todo lo que puede pasar. } \\
\text { e ideas. } \\
\text { de investigación. } \\
\text { nbros del grupo. }\end{array}$ \\
\hline & ACTIVIDADES \\
\hline LLUVIA DE IDEAS & Cada persona propone uno o varios temas sobre los que se quiere trabajar. \\
\hline ESCOGER TEMA & $\begin{array}{l}\text { Se selecciona la propuesta que más nos interesa. Qué tema se quiere } \\
\text { representar. ¿Qué se quiere transmitir? }\end{array}$ \\
\hline INVESTIGACIÓN & $\begin{array}{l}\text { Búsqueda de documentación sobre el tema elegido. } \\
\text { Aprendemos sobre el tema. } \\
\text { Buscamos referencias en música, películas, libros, periódicos o pinturas. }\end{array}$ \\
\hline $\begin{array}{l}\text { EMOCIONES } \\
\text { EXPERIMENTADAS }\end{array}$ & $\begin{array}{l}\text { Indagación personal sobre la reacción emocional que nos genera el tema } \\
\text { elegido. }\end{array}$ \\
\hline
\end{tabular}


Continuación de lo cuadro 1

\begin{tabular}{|c|c|}
\hline $\begin{array}{l}\text { ACCIONES } \\
\text { ESPONTÁNEAS }\end{array}$ & $\begin{array}{l}\text { A partir del material seleccionado realizaremos exploraciones e } \\
\text { improvisaciones, individuales y grupales. Qué respuestas motrices nos } \\
\text { suscita el tema. }\end{array}$ \\
\hline $\begin{array}{l}\text { REFLEXIÓN, } \\
\text { VALORACIÓN }\end{array}$ & $\begin{array}{l}\text { Descripción de las vivencias suscitadas en cada una de las actividades } \\
\text { de esta fase. }\end{array}$ \\
\hline \multicolumn{2}{|l|}{ FASE II. Composición } \\
\hline \multicolumn{2}{|c|}{$\begin{array}{l}\text { - Fase en la que se empieza a componer. } \\
\text { - Se van tanteando las propuestas individuales y grupales } \\
\text { - Se mezclan, y se toman decisiones de cuándo y qué utiliz } \\
\text { aquello que nos gusta y desechamos lo que creemos que } \\
\text { - Es un momento para aportar un abanico de propuestas. } \\
\text { - Dejaremos reposar las ideas que van surgiendo. }\end{array}$} \\
\hline \multicolumn{2}{|c|}{ ACTIVIDADES } \\
\hline \multirow[t]{3}{*}{$\begin{array}{l}\text { SELECCIÓN DE } \\
\text { PROPUESTAS }\end{array}$} & $\begin{array}{l}\text { Seleccionar las improvisaciones que nos gustan y juntar las propuestas } \\
\text { prácticas de todos los miembros del grupo. } \\
\text { Modificarlas, mejorarlas a partir de los criterios: calidad en la expresión } \\
\text { y uso del cuerpo, espacio, tiempo, interacciones. La originalidad. La } \\
\text { claridad en el mensaje. La capacidad de provocar emociones. }\end{array}$ \\
\hline & $\begin{array}{l}\text { Realizar la composición a partir de un hilo conductor: inicio-nudo-desenlace } \\
\text { Primer esbozo de la escena. }\end{array}$ \\
\hline & Representamos gráficamente las distintas escenas. \\
\hline COMPOSICIÓN & $\begin{array}{l}\text { Describimos sus partes en cada uno de los momentos: inicio-nudo- } \\
\text { desenlace. Detallando el espacio utilizado, las direcciones, trayectorias. } \\
\text { Qué participantes entran en escena. El tiempo que duran. La música } \\
\text { utilizada, si es el caso. Vestuario seleccionado, entre otros aspectos. }\end{array}$ \\
\hline $\begin{array}{l}\text { REFLEXIÓN, } \\
\text { VALORACIÓN }\end{array}$ & $\begin{array}{l}\text { Descripción de las vivencias suscitadas en cada una de las actividades } \\
\text { de esta fase. }\end{array}$ \\
\hline \multicolumn{2}{|l|}{ FASE III: Retoque } \\
\hline \multirow{2}{*}{\multicolumn{2}{|c|}{$\begin{array}{l}\text { - Realizamos una primera actuación con la composición creada } \\
\text { - Miramos críticamente lo que hemos creado y hacemos los retoques pertinentes. } \\
\text { - Damos el formato final a la composición. }\end{array}$}} \\
\hline & \\
\hline \multirow[b]{2}{*}{ RETOQUES } & $\begin{array}{l}\text { Con la primera composición elaborada, que es como un boceto, } \\
\text { realizamos una grabación en vídeo para poder valorar nuestro trabajo. }\end{array}$ \\
\hline & $\begin{array}{l}\text { Se dan soluciones a lo que no funciona y se añaden los cambios } \\
\text { pertinentes. Podemos observar y mejorar a partir de los siguientes criterios: } \\
\text { CUERPO } \\
\text { Adecuación de la expresión facial al personaje / situación } \\
\text { Expresividad global: "Me creo el papel", "estoy presente" } \\
\text { Muestro dominio corporal: uso de tensiones, suspensiones, caídas, } \\
\text { equilibrios ... } \\
\text { ESPACIO } \\
\text { Variación en el uso de los niveles (alto, medio y bajo) } \\
\text { Buena orientación respecto el foco } \\
\text { Las direcciones utilizadas son claras } \\
\text { TIEMPO } \\
\text { Utilizamos los silencios, los acentos para aumentar la expresividad } \\
\text { Diversidad en el uso tempo (diferentes velocidades, acción no monótona) } \\
\text { PARTICIPANTES } \\
\text { Me coordino con los compañeros / as } \\
\text { Originalidad en las interacciones realizadas } \\
\text { MATERIALES (ropa, objetos, efectos de sonido, música) } \\
\text { Adecuado a lo que queremos transmitir } \\
\text { COMUNICACIÓN } \\
\text { Transmitimos un mensaje } \\
\text { Hacemos emocionar al público } \\
\text { CREATIVIDAD } \\
\text { Originalidad en el modo de transmitir el mensaje }\end{array}$ \\
\hline
\end{tabular}

Continúa en la página siguiente... 
Continuación de lo cuadro 1

\begin{tabular}{|c|c|}
\hline $\begin{array}{l}\text { REFLEXIÓN, } \\
\text { VALORACIÓN }\end{array}$ & $\begin{array}{l}\text { Descripción de las vivencias suscitadas en cada una de las actividades } \\
\text { de esta fase. }\end{array}$ \\
\hline \multicolumn{2}{|l|}{ FASE IV: Muestra final } \\
\hline \multicolumn{2}{|c|}{$\begin{array}{l}\text { - Puesta en escena final. } \\
\text { - La respuesta del público da el feedback para saber qué acogida tiene lo producido. }\end{array}$} \\
\hline & ACTIVIDADES \\
\hline ESCENIFICACIÓN & $\begin{array}{l}\text { Escenificar ante el público / clase, el proyecto elaborado. } \\
\text { Presentamos la composición con un título. } \\
\text { Se presenta una sinopsis de la obra. } \\
\text { Preparamos todos los recursos necesarios para la actuación. }\end{array}$ \\
\hline FEEDBACK & Captamos los aplausos del público \\
\hline $\begin{array}{l}\text { REFLEXIÓN, } \\
\text { VALORACIÓN }\end{array}$ & $\begin{array}{l}\text { Descripción de las vivencias suscitadas en cada una de las actividades de } \\
\text { esta fase. }\end{array}$ \\
\hline
\end{tabular}

Fuente: elaboración propia

\subsection{INSTRUMENTOS}

La recogida de las experiencias del alumnado se realizó a través de un informe grupal que fue cumplimentado por los participantes que formaban parte de los grupos de creación. Cada grupo rellenó los apartados que aparecen en el Cuadro 1 y los entregaron a la profesora una vez finalizado el proceso. Los informes fueron escritos en catalán, pero los fragmentos transcritos en el apartado de resultados han sido traducidos al castellano.

Para analizar las percepciones registradas se utilizó el análisis de contenido. En la Tabla 1 se presenta el sistema de categorías construido para poder efectuar el análisis.

Tabla 1 - Sistema de categorías

\begin{tabular}{lll}
\hline \multicolumn{1}{c}{ Dimensión } & \multicolumn{1}{c}{ Categorías } & \multicolumn{1}{c}{ Indicadores } \\
\hline & & \\
& & 1.1 .1$. Consenso \\
& 1.1.Fase 1 Lluvia de ideas & 1.1.2. Familiaridad \\
1. Interacción & & 1.1 .3$. Cohesión grupal \\
social en los & & 1.1 .4$. Implicación \\
\cline { 2 - 3 } procesos de & & 1.2 .1$. Consenso \\
creación grupal & \multirow{2}{*}{ 1.2. Fase 2 Composición } & 1.2.2. Familiaridad \\
& & 1.2 .3$. Cohesión grupal \\
& & 1.2 .4$. Implicación \\
& & 1.2 .5$. Asignación roles \\
\hline
\end{tabular}

Fuente: elaboración propia

Este sistema de categorías identifica los factores de la interacción social que dificultan y facilitan el proceso de creación. Hay una única dimensión que es la interacción social en los procesos de creación grupal. Las dos categorías obedecen a dos de las cuatro fases del proceso de creación, más concretamente las dos primeras -fase 1 Lluvia de ideas y fase 2 Composición-, ya que, en las otras dos no aparecieron registros vinculados con la interacción social. Los indicadores son los diferentes aspectos que concretan la interacción social. 
Para su elaboración se siguió un proceso inductivo y deductivo, ya que se parte de un modelo teórico sobre el objeto de estudio que es modelado ad hoc adaptándose a los testimonios de los informantes y a la coherencia de la intervención.

El sistema de categorías elaborado cumple los requisitos planteados por Heinemann (2003). En primer lugar, se adecua al objeto de estudio, es decir, refleja las intenciones de la búsqueda. En segundo lugar, sus categorías e indicadores satisfacen los criterios de exhaustividad y de mutua exclusividad. Finalmente, la objetividad y fidelidad del sistema permite que los fragmentos de un mismo material puedan ser codificados de la misma manera por distintos analistas.

\subsection{PROCEDIMIENTO}

El informe grupal se cumplimentó al final del proceso de creación, más concretamente, después de la puesta en escena. Este informe formó parte de la evaluación de la asignatura. Se calificó la cumplimentación de los apartados del informe por parte de los estudiantes.

Posteriormente se aplicaron las tres fases propuestas por Bardin (2002): preanálisis, explotación del material y tratamiento e interpretación de datos.

En la fase preanálisis se confeccionó el sistema de categorías. Para ello, se realizó una lectura superficial de los informes grupales y se propuso un borrador. A continuación se llevó a cabo una prueba piloto sobre una submuestra seleccionada al azar (30\% de la muestra).

Seguida de la prueba piloto se llevó a cabo un pretest de fiabilidad en un cálculo de acuerdo entre dos codificadoras independientes, siendo codificados el $20 \%$ de los informes. Previamente las codificadoras se sometieron a un proceso de entrenamiento de dos horas para conseguir máxima precisión, calibre y consistencia en su codificación. Inicialmente se les explicó el objetivo de estudio, se presentó el sistema de categorías y se les entregó las definiciones de cada una de las categorías e indicadores para facilitar la comprensión del sistema. Las dos codificadoras tenían amplia experiencia en el análisis de contenido. Se utilizó el coeficiente de kappa de Cohen como medida de concordancia, teniendo buena concordancia $(\mathrm{k}=.780)$.

En la explotación del material se codificaron todos los informes de forma consensuada por el equipo de investigación. En primer lugar, se seleccionaron los fragmentos a codificar y posteriormente se procedió uno a uno. Se dejó un tiempo de reflexión individual y luego se exponía la selección del indicador y el argumento de dicha decisión. En caso de no existir coincidencia se iniciaba un debate basado en las definiciones del sistema de categorías.

El tratamiento e interpretación de los resultados se llevó a cabo utilizando el programa informático QSR-NVIVO 11, diseñado para el análisis de datos en investigaciones basadas en métodos cualitativos.

Consideraciones éticas: Un miembro del grupo de investigación fue la profesora de la asignatura, y por lo tanto, se debe de tener en cuenta la influencia 
profesora-alumno/a. Esta relación también implica un exhaustivo conocimiento sobre el proceso de creación, facilitando la resolución de cualquier problema que haya surgido en la investigación (ELIOT, 2000).

Los participantes recibieron información sobre el objetivo de la investigación, así como, dieron su permiso para la utilización de los informes grupales.

\section{RESULTADOS}

Se han codificado un total de 111 referencias, 64 pertenecientes a la fase 1 Lluvia de ideas $(57,7 \%)$ y 47 a la fase 2 Composición (42,3\%). Todas las referencias menos cuatro $(96,4 \%)$ describen de forma positiva como la interacción social facilita el proceso de creación. Es decir, la interacción social es un facilitador para los procesos de creación grupal.

En la Figura 1 se ha detallado el número de referencias según el indicador y la fase del proceso de creación. Lo más característico de este gráfico es que los indicadores del consenso son los más numerosos con gran diferencia (52 referencias de consenso en la fase 1 -indicador 1.1.1. - y 25 referencias en la fase 2 -indicador 1.2.1. -$)$.

Figura 1 - Número de referencias por indicador y fase del proceso de creación

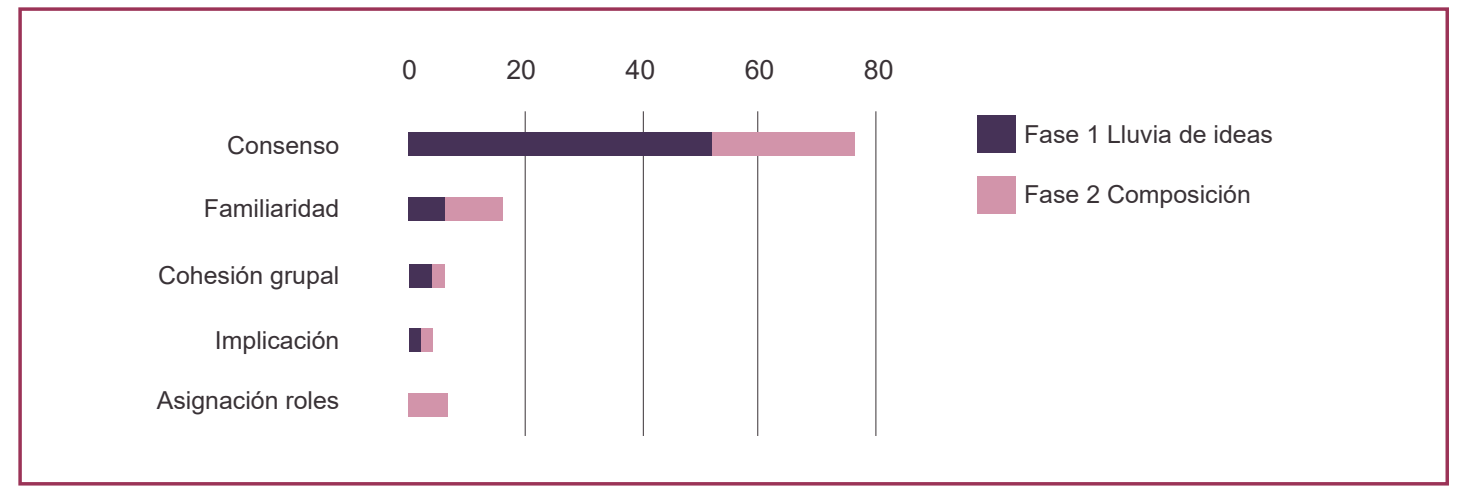

Fuente: elaboración propia

\subsection{EL CONSENSO COMO FACILITADOR EN LOS PROCESOS DE CREACIÓN GRUPAL}

El consenso se recoge en dos indicadores (1.1.1. y 1.2.1.), cada uno ubicado en una fase diferente del proceso de creación. Estos indicadores juntos suman 77 referencias, y suponen casi el $70 \%$ del total de las referencias del estudio.

El consenso es entendido como el acuerdo producido por consentimiento entre todos los miembros de un grupo. Se debe de recordar que la primera fase Lluvia de ideas se inicia con una lluvia de ideas que se concreta en la elección de un tema. La segunda fase Composición se distingue porque se organizan y estructuran las ideas, así como, se asignan los roles a los miembros del grupo.

En la Figura 2 se pueden distinguir las diferentes unidades de contenido que conforman el consenso. Lo más destacable es que el proceso democráticoasambleario es el más citado, teniendo una cobertura del $48 \%$ del total de consenso. 
También se debe destacar que sólo hay cuatro referencias que aluden a la falta de consenso (disenso), todas las demás (73 referencias de 77 , casi el $95 \%$ ) describen el acuerdo establecido en la toma de decisiones en las dos fases.

Figura 2 - Número de referencias de las unidades de contenido del consenso según las fases de creación

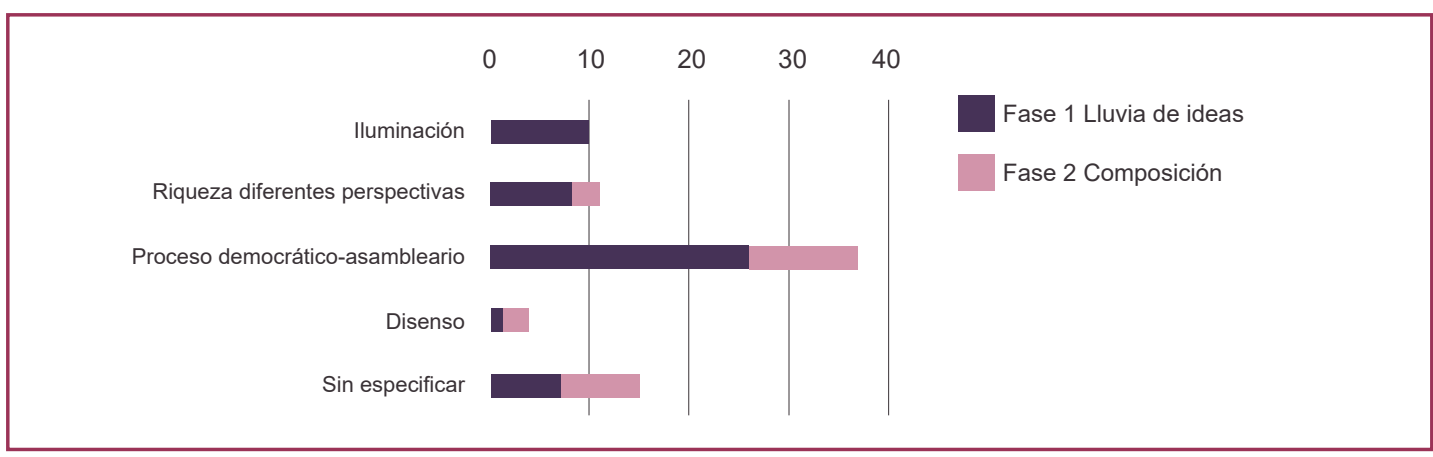

Fuente: elaboración propia

Como se comentaba con anterioridad, el proceso democrático-asambleario es la unidad de contenido con mayor número de referencias ( $48 \%$ del total de consenso). Se refiere a la participación de todos los integrantes del grupo en la aportación de ideas. Por ejemplo G. expone:

Considero que fue una parte fundamental porque cada uno de nosotros aportaba su pequeño grano de arena para poder encauzar el proyecto hacia un espacio en el que todos nos sintiésemos cómodos. Esto posibilitó que el grupo estuviese mucho más unido.

También C. describe la facilidad a la hora de escoger el tema: “(...) nos resultó muy fácil coincidir en el tema a escoger. En nuestro caso cada uno propuso un tema, y entre todos votamos el que nos gustaba más".

La segunda unidad de contenido con mayor número de referencias es la riqueza percibida por la diversidad de perspectivas ante un mismo fenómeno. El hecho de crear de forma grupal hace que se aporten ideas totalmente diversas y eso enriquece el proceso. Por ejemplo R. así lo describe: "Fase muy interesante y divertida, ya que se pudo observar una gran variedad de ideas, la mayoría muy curiosas, de las cuales, podrían resultar muy divertidas combinándolas con otras propuestas".

La iluminación sólo aparece en la fase 1 Lluvia de ideas, y se refiere a la luminosidad que ha ejercido en el grupo una de las ideas surgidas y que ha posibilitado el consenso de forma inmediata por parte de todos los miembros: "Estuvimos un buen rato buscando ideas, pero cuando salió el tema de la marginación a un sin techo, ninguno dudó de que era un tema ideal" (A.).

Con respecto al disenso, la única referencia en negativo de la fase 1 Lluvia de ideas describe el malestar experimentado por un alumno: "He experimentado frustración y rabia al sentir que en ocasiones mis ideas no eran escuchadas, y las rechazaban sin dejarme expresarlas. Al ser un trabajo en grupo tenía que ser en conjunto (J.)".

Las otras tres referencias están ubicadas en la segunda fase y pertenecen a tres participantes de grupos diferentes. Todos los casos coinciden en describir esta 
fase como la más compleja y caótica, de ahí que resultase muy difícil aunar todas las opiniones y perspectivas del proceso de creación. S. es muy explícito:

Por lo que respecta en la fase dos: fue la más caótica porque estábamos creando las ideas previas en escenas y no parábamos de modificar y parar 20 veces en un mismo acto. Eso generó diversidad de opiniones y comportamientos respecto a las ideas propuestas.

\subsection{LOS OTROS INDICADORES DEL PROCESO DE CREACIÓN GRUPAL. FAMILIARIDAD, COHESIÓN GRUPAL, IMPLICACIÓN Y ASIGNACIÓN DE ROLES}

La familiaridad con un total de 16 referencias ( $14 \%$ del total de referencias) se entiende como el sentimiento de confianza que se tiene con los compañeros/as del grupo. La presencia del grupo respalda las acciones individuales, facilitando de esta manera la lluvia de ideas en la fase 1: "Me he sentido cohibida, ya que muchas de las ideas que proponía no gustaban a nadie. Pero a medida que íbamos avanzando me sentía mucho más reforzada por mis compañeros (L.)".

Las referencias de la fase 2 describen la ayuda que ofrece el grupo en los momentos de escasa lucidez que pueden tener en ocasiones algunas personas: "No me he sentido inspirada en la creación de escenas, y por tanto, se puede decir que el trabajo lo han realizado mis compañeros" (M.). También ubicadas en esta fase 2 algunas referencias destacan que la fluidez del proceso de creación está determinada porque el grupo está consolidado al conocerse desde varios meses: "(...) y como ya nos habíamos conocido durante el curso, las diferentes escenas surgieron de manera fluida entre todos" (I.).

La asignación de roles sólo ha sido registrada en la fase 2 Composición, ya que, esta acción es inexistente en la fase anterior. Se han registrado siete referencias $(6 \%)$, y tres de ellas describen la comodidad y fluidez que supuso este proceso, y es que:

[...] en esta parte hicimos una elección del personaje que haría cada persona y quien se adaptaría mejor. Fue un momento bastante entretenido, ya que todos dábamos ideas de cómo hacer cada personaje y crearle una personalidad (A.).

Otra referencia explica que el criterio de la asignación está vinculado con la personalidad de cada miembro del grupo: "Nos hemos basado en la personalidad de cada uno para repartir los personajes, y fuimos decidiendo cada personaje sí todos estábamos de acuerdo, y así fue" (S.). Con respecto al personaje asignado, I. manifiesta el reto que le supuso representar el personaje más importante de la obra:

Al comienzo me asignaron un personaje de tanto peso como el protagonista del proceso de creación y fue muy impactante, pero a la vez ha supuesto todo un reto personal. Creo que este proyecto me ayudará a desarrollarme como un profesional y conseguiré tener una mejor imagen de mí.

Por último, en este apartado, P. comparte su preferencia por adoptar un personaje u otro de la obra:" Lo que tenía claro desde el comienzo es que quería ser republicana, y no quería hacer el papel de facha, ya que no me sentiría identificada ni cómoda al ponerme en el papel del personaje". 
La cohesión grupal supone un 5\% (seis referencias) del total. Estas referencias describen como el grado de adhesión de los miembros del grupo facilita el proceso de creación.

Por último, la implicación sólo tiene registradas cuatro referencias $(3 \%)$, las cuales establecen el alto grado de compromiso que es clave para poder trabajar en grupo.

\section{DISCUSIÓN}

Los resultados de la presente investigación demuestran que la interacción social facilita el proceso de creación, ya que las referencias codificadas detallan los aspectos positivos de la interacción social como facilitadora del proceso de creación grupal. Estos datos coinciden con estudios previos desarrollados en educación superior en los que se manifestaba la importancia de la interacción social en la creación grupal (HAN; HAN; BRASS, 2014; LIU, 2017).

Las tareas artístico-expresivas se caracterizan por fomentar el trabajo de la singularidad, lo que provoca que emerjan las carencias en el alumnado ante la falta de práctica al enfrentarse a los procesos de creación y un miedo a la inexistencia de un modelo que paraliza la fluidez de respuestas (SIERRA, 2002). Por este motivo, este estudio refleja la puesta en práctica de un programa de intervención de expresión corporal y danza fundamentado en los procesos de creación grupal que sigue la indicación de Giguere (2011) de plantear propuestas pedagógicas creativas de pensamiento grupal para contrarrestar dichas carencias y facilitar los procesos de creación.

Otro de los resultados de la presente investigación desvelan que el consenso es el factor de la interacción social más considerado por su valor en el proceso de creación. Los participantes describen el consenso como facilitador del proceso de creación, datos que coinciden con el estudio de Torrents et al. (2008) en el que se resaltaba la importancia del consenso entre los miembros del grupo para compensar las divergencias creativas.

Dentro del consenso, los testimonios refieren como principal elemento facilitador el proceso democrático-asambleario, ya que permite el acuerdo consensuado entre todos los miembros del grupo tras la aportación de ideas. Estos resultados confirman el rol facilitador de los participantes en el proceso de creación grupal (GIGUERE, 2011), para el cual, se necesitan requerimientos sociales como escucha, comunicación, apertura y asunción de riesgos (RYMER, 2017).

La riqueza percibida por la diversidad de ideas y la iluminación surgida ante una idea propuesta por el grupo, son percibidas por los participantes como positivas en el proceso de creación. Esta influencia positiva de la diversidad del capital social en la creatividad corrobora el estudio de Han, Han y Brass (2014) y se confirma que las propuestas de creación en grupo generan sentimiento comunitario y mejoran la eficacia individual y colectiva (REMESAL; COLOMINA, 2013).

Aunque la falta de acuerdo -disenso- ha sido uno de los factores que menos se ha manifestado en los testimonios de los participantes, es importante mencionarlo 
como un factor que dificulta los procesos de creación grupal. Los estudiantes mencionan el disenso durante la fase lluvia de ideas al no sentirse escuchados y en la fase composición por la dificultad que suponía aunar todas las opiniones del proceso de creación. Estos datos nos indican la importancia de incorporar en la enseñanza universitaria propuestas educativas colaborativas que desarrollen las habilidades sociales (ARRIBAS-GARRALAGA et al., 2019; MONZONÍS, 2015) y eviten las conductas de exclusión, marginación o conflicto propias de los modelos más tradicionales de educación física (CAPLLONCH; FIGUERAS; CASTRO, 2018).

Otro de los indicadores que facilitan el proceso de creación manifestados en los discursos de los participantes es la familiaridad, reflejándose ésta en el sentimiento de confianza que se tiene con los compañeros/as del grupo y la ayuda del mismo ante la falta de ideas en la fase de composición. Estos datos coinciden con las investigaciones que vinculan los climas sociales positivos con un mejor afecto (STARK; NEWTON, 2014), la confianza como requerimiento social para colaborar creativamente (RYMER, 2017) y la importancia de pedir ayuda (PÉREZALBARRACÍN; FERNÁNDEZ-BAENA, 2019) para la resolución de conflictos.

Por último, los estudiantes también manifiestan la cohesión grupal, la implicación y la asignación de roles como elementos que favorecen los procesos de creación grupal. La cohesión grupal se refleja en los testimonios a través del grado de adhesión de los miembros, lo que concuerda con los lazos de colaboración expuestos por Zander et al. (2014). La implicación referida al alto grado de compromiso en el proceso también fue evidenciada previamente por Arribas-Garralaga et al. (2019). La asignación de roles relacionada con la comodidad o fluidez o el criterio de la personalidad de cada miembro para el reparto de personajes indica que los procesos de creación grupales ocurren en un contexto social que da lugar a un énfasis en las dimensiones centradas en el grupo y, a su vez, generan sentimiento comunitario y dinámicas de relaciones positivas (REMESAL; COLOMINA, 2013).

\section{CONCLUSIONES}

Tras el análisis de los resultados del presente estudio se puede concluir que:

- La interacción social facilita los procesos de creación grupales propuestos en el curso de danza y expresión corporal. Los principales elementos facilitadores son el consenso y la familiaridad.

- El proceso democrático-asambleario en la aportación de ideas, la riqueza percibida por la diversidad de ideas y la iluminación surgida ante una aportación al grupo, son los factores de consenso que facilitan los procesos de creación. Por otra parte, la falta de consenso es provocada cuando un miembro del grupo no se siente escuchado o ante la dificultad de aunar todas las opiniones en la fase de creación.

- La familiaridad se expone de dos maneras: en el sentimiento de confianza que se tiene con los compañeros/as del grupo y la ayuda del mismo ante la falta de ideas en la fase de composición.

- Otros elementos que favorecen los procesos de creación grupal son la cohesión grupal, la asignación de roles y la implicación de los miembros. 
Para finalizar, se sugiere utilizar como recurso pedagógico, el trabajo en procesos de creación grupal en actividades artístico-expresivas en el desarrollo de las habilidades sociales para la resolución de conflictos entre los estudiantes universitarios.

\section{REFERENCIAS}

ARRIBAS-GALARRAGA, Silvia; LUIS-DE COS, Izaskun; LUIS-DE COS, Gurutze; URRUTIA-GUTIÉREZ, Saioa. Aprendizaje cooperativo: un proyecto de expresión corporal en el grado de Educación Primaria. Journal of Sport and Health Research, v. 11, supl. 1, p.155-166, 2019. Disponible en: http://www.journalshr.com/papers/Vol\%2011 suplemento/ JSHR\%20V11 supl 01 14.pdf. Acceso en: 5 ene.2020.

BANNERMAN, Christopher. Creativity and Wisdom. In: CRAFT, Anna; GARDNER, Howard; CLAXTON, Guy. Creativity, Wisdom, and Trustee-Ship. Thousand Oaks, CA: Corwim Press, 2008. p. 133-142.

BARDIN, Laurence. Análisis de contenido. Madrid: Akal, 2002.

CAMACHO-MIÑANO, María José; PRAT, María. Violencia simbólica en la educación física escolar: un análisis crítico de las experiencias negativas del futuro profesorado de educación primaria. Movimento, v. 24 , n. 3, p. 815-826, 2018.

CAPLLONCH, Marta; FIGUERAS, Sara; CASTRO, Marcos. Estrategias para la resolución de conflictos en educación física. Apunts, v. 133, p. 50-67, 2018. Disponible en: https:// hemeroteca.revista-apunts.com/apunts/articulos//133/es/050-067.pdf. Acceso en: 14 feb. 2020.

ELLIOT, John. El cambio educativo desde la investigación-acción. Madrid: Morata, 2000.

GIGUERE, Miriam. Dancing thoughts: an examination of children's cognition and creative process in dance. Research in Dance Education, v. 12, n. 1, p. 5-28, 2011.

HAN, Jing; HAN, Jian; BRASS, Daniel. Human capital diversity in the creation of social capital for team creativity. Journal of Organizational Behavior, v. 35, p. 54-71, 2014.

HEINEMANN, Klaus. Introducción a la metodología de la investigación empírica. Barcelona: Paidotribo. 2003.

LIU, Chih-Hsing Sam. Remodelling Progress in Tourism and Hospitality Students' Creativity through Social Capital and Transformational Leadership. Journal of Hospitality, Leisure, Sport \& Tourism Education, v. 21, p. 69-82, 2017. DOI: https://doi.org/10.1016/j. ihlste.2017.08.003

MOLINA, Fidel. Nuevos conflictos sociales y su presencia educativa. Análisis sociológico y reflexiones para la intervención. Cultura y Educación, v. 17, n. 3, p. 17-28, 2005.

MOLINA, Fidel. Teaching in physical education: socialization, play and emotions. Electronic Journal of Research in Educational Psychology, v. 10, n. 2, p. 755-770, 2012.

MOLL, Ashley; LAMBERT, Susan; VISKER, Josepg; DUNSEITH, Nicole; WANG, Anna; AZIM, Sabiya; COX, Carolyn. A case study activity to assess nursing students' perceptions of their inter professional healthcare team's collaborative decision-making process. Journal of Interprofessional Education \& Practice, v. 14, p. 18-21, 2019. 
MONZONÍS, Nuria. La educación física como elemento de mejora de la competencia social y ciudadana. Tesis (doctoral). Barcelona: Universidad de Barcelona. 2015. Disponible en: https://www.tdx.cat/bitstream/handle/10803/370837/NMM 1 de2. pdf? sequence=1\&isAllowed=y. Acceso en: 23 feb. 2020.

PÉREZ-ALBARRACIN, Ana; FERNÁNDEZ-BAENA, Javier. Más allá de la resolución de conflictos: Promoción de aprendizajes socioemocionales en el alumnado mediador [Beyond conflict resolution: socio-emocional Learning in student mediators]. Electronic journal of research in education psychology, v. 17, n. 2, p. 355-358, 2019. Disponible en: https:// pdfs.semanticscholar.org/9caf/d906e5c74d34f8c5caa45bec4920b9cb7438.pdf. Acceso en: 1 mar. 2020.

REMESAL, Ana; COLOMINA, Rosa. Social presence and online collaborative small grupo work: A socioconstructivist account. Computers \& Education, v. 60, p. 357-367, 2013.

ROJAS-RUIZ, Gloria; ALEMANY-ARREBOLA, Inmaculada. Perception of conflict in university students. The importance of initial training. Cultura y Educación, v. 28, n. 2, p. 269-300, 2016.

ROVIRA, Gloria. Danza y expresión corporal. Vitoria-Gasteiz: Bradu 20 SL., 2019.

RYMER, Jess. An argument for investigation into Collaborative, Choreomusical Reltionships within Contemporary Performance: A Practical and Theoretical Enquiry into the Distinct Contributions of A Collaborative, Co-creative Approach. Avant Trends in Interdisciplinary Studies, v. 7, p. 181-196, 2017.

SÁEZ DE OCÁRIZ, Unai; LAVEGA, Pere. Transformar conflictos en educación física en primaria a través del juego. Aplicación de índice de conflictividad. Cultura y Educación, v. 25, n. 4, p. 549-560, 2013.

SÁEZ DE OCÁRIZ, Unai; SERNA, Jorge; LAVEGA, Pere; COSTES, Antoni; MARCH, Jaume. Coeducación y experiencias positivas. Aportaciones a la convivencia desde la expresión motriz cooperativa. Movimento, v. 23, n. 3, p. 1053-1064, 2017.

SERRAT, Albert (coord.) Resolución de conflictos: una perspectiva globalizadora. Barcelona: Cisspraxis, 2002.

SHEPHERD, Hana. The Structure or Perception: How Networks Shape Ideas of Norms. Sociological Forum, v. 32, n. 1, p. 72-93, 2017.

SIERRA, Miguel Ángel. La expresión corporal desde la perspectiva del alumnado de Educación Física. Madrid: Universidad Complutense de Madrid, 2002.

STARK, Andrea; NEWTON, Maria. A dance's well-being: The influence of the social psychological climate during adolescence. Psychology of Sport and Exercise, v. 15, p. 356-363, 2014.

TORRENTS, Carlota; CASTAÑER, Marta; DINUSOVA, María; ANGUERA, María Teresa.T. El efecto del modelo docente y de la interacción con compañeros en las habilidades motrices creativas de la Danza. Un formato de campo para su análisis y obtención de T-patterns motrices. Retos, v. 14, p. 5-9, 2008.

ZANDER, Lysann; KREUTZMANN, Madeleine; WEST, Stephen; METTKE, Ellen; HANNOVER, Bettina. How school-based dancing classes change affective and collaborative networks of adolescents. Psychology of Sport and Exercise, v. 15, p. 418-428, 2014. 
Resumo: Este artigo identifica os fatores da interação social que facilitam e dificultam o processo de criação grupal para a elaboração de uma composição cênica. A mostra está formada por 105 estudantes universitarios - sendo 85 homens e 20 mulheres - com uma média de idade de $19.99 \pm 2.78$ anos e relacionados à Faculdade de Ciências da Atividade Física e Esporte em EUSES, Universidade de Girona (Espanha). Cada grupo elaborou um informe sobre o processo de criação no qual se demonstravam as dificuldades e facilidades frente tal composição durante as sessões práticas da matéria de Dança e Expressão Corporal. Posteriormente se realizou a análise de conteúdo de tais informes grupais. Para o tratamento dos dados foi utilizado o Nvivo 11. Os resultados mostraram que: a) a interação social facilita o processo de criação grupal; b) o consenso é o principal fator da interação social que facilita o processo de criação grupal.

Palavras chave: Dança. Consenso. Relações Interpessoais. Criatividade.

Abstract: This article points out social interaction factors that facilitate and hinder the group creation process for designing a scenic composition. The study's sample comprised 105 university students -85 males and 20 females - aged $19.99 \pm 2.78$ on average, from the bachelor's degree in Sciences of Physical Activity and Sport at EUSES, the University of Girona (Spain). Each group prepared a report on the creation process in which they reflected on those factors that facilitated or hindered said composition during the practical sessions of the subject Dance and Bodily Expression. Subsequently, content analysis was carried out on the group's reports and Nvivo 11 software was used to process the data. The results showed that a) social interaction facilitates the group creation process and b) consensus is the main social interaction factor that facilitates it.

Keywords: Dancing. Consensus. Interpersonal relations. Creativity. 


\section{LICENCIA DE USO}

Este es un artículo publicado en Open Access bajo la licencia Creative Commons Atribución 4.0 Internacional (CC BY 4.0), que permite su uso, distribución y reproducción en cualquier medio, siempre que la obra original esté correctamente citada. Más información en: http://creativecommons.org/licenses/by-nc/4.0

\section{CONFLICTO DE INTERESES}

Los autores han declarado que no existe conflicto de intereses en este trabajo.

\section{CONTRIBUCIONES DE LOS AUTORES}

Inma Canales-Lacruz: análisis e interpretación de los datos, redacción del resumen, método y resultados.

Alba González-Palomares: análisis e interpretación de los datos, redacción de la introducción, discusión y conclusiones.

Gloria Rovira: diseño del proceso de creación, búsqueda bibliográfica, revisión del manuscrito, recogida de datos y análisis de los datos.

\section{FINANCIACIÓN}

El presente trabajo se llevó a cabo sin ningún apoyo financiero.

\section{CÓMO HACER REFERENCIA}

CANALES-LACRUZ, Inma; GONZÁLEZ-PALOMARES, Alba; ROVIRA, Gloria.

El proceso de creación grupal en las actividades artístico-expresivas como escenario para la negociación y la resolución de conflictos. Movimento (Porto Alegre), v.26, p. e26098, jan./dez. 2020. Disponible en: https://seer.ufrgs.br/ Movimento/article/view/105472. Acceso en: [día] [mes abreviado]. [año]. DOI: https://doi.org/10.22456/1982-8918.105472

\section{RESPONSABILIDAD EDITORIAL}

Alex Branco Fraga*, Elisandro Schultz Wittizorecki*, Ivone Job*, Mauro Myskiw*, Raquel da Silveira*

*Universidade Federal do Rio Grande do Sul, Escola de Educação Física, Fisioterapia e Dança, Porto Alegre, RS, Brasil 